\title{
Author Index Volume 3 (2016)
}

Abeling, T., see Pelling, M.

3 (2016) 1650012

Agboola, J., see Ajibade, I.

3 (2016) 1650009

Agboola, J. I., see Birkmann, J.

3 (2016) 1650019

Ahove, M., see Birkmann, J.

3 (2016) 1650019

Ajibade, I., Pelling, M., Agboola, J. \& Garschagen, M., Sustainability Transitions: Exploring Risk Management and the Future of Adaptation in the Megacity of Lagos

3 (2016) 1650009

Barnes, M., His Work/Our Work: Advancing the Learning Values of James Kenneth Mitchell's Hazards in Context Framework

2 (2016) 1671008

Berchtold, C., see Schwab, M.

4 (2016) 1650015

Birkmann, J., Agboola, J. I., Welle, T., Ahove, M., Odunuga, S., von Streit, J. \& Pelling, M., Vulnerability, Resilience and Transformation of Urban Areas in the Coastal Megacity Lagos: Findings of Local Assessments and a Household Survey in Highly Exposed Areas

3 (2016) 1650019

Birkmann, J., see Welle, T.

3 (2016) 1650018

Birkmann, J. \& Welle, T., The WorldRiskIndex 2016: Reveals the Necessity for Regional Cooperation in Vulnerability Reduction Birkmann, J., Wenzel, F., Greiving, S., Garschagen, M., Vallée, D., Nowak, W., Welle, T., Fina, S., Goris, A., Rilling, B., Fiedrich, F., Fekete, A., Cutter, S. L., Düzgün, S., Ley, A., Friedrich, M., Kuhlmann, U., Novák, B., Wieprecht, S., Riegel, C., Thieken, A., Rhyner, J., Ulbrich, U. \& Mitchell, J. K., Extreme Events, Critical Infrastructures, Human Vulnerability and Strategic Planning: Emerging Research Issues

4 (2016) 1650017

Bosma, K., see Douglas, E. M.

4 (2016) 1650013

Camargo, S. J., see Sobel, A.

1 (2016) 1671001

Catalano de Sousa, M. R., Montalto, F. A. \& Gurian, P., Evaluating Green Infrastructure Stormwater Capture

Performance Under Extreme Precipitation

2 (2016) 1650006 
Chatterjee, M., Advancing Climate Change Adaptation:

Borrowing from the Natural Hazard Research (NHR) Tradition in Geography

2 (2016) 1602001

Clarke, D., Murphy, C. \& Lorenzoni, I., Barriers to

Transformative Adaptation: Responses to Flood Risk in Ireland

2 (2016) 1650010

Cutter, S. L., see Birkmann, J.

4 (2016) 1650017

Cutter, S. L., The Changing Context of Hazard Extremes: Events, Impacts, and Consequences

2 (2016) 1671005

Debucquoy, W., see Sobel, A.

1 (2016) 1671001

Deodatis, G., see Sobel, A.

1 (2016) 1671001

Douglas, E. M., Kirshen, P. H., Bosma, K., Watson, C., Miller, S. \& Mcarthur, K., Simulating the Impacts and Assessing the Vulnerability of the Central Artery/Tunnel System to Sea Level Rise and Increased Coastal Flooding

Du Plessis, R., see Gibson, T. D.

Düzgün, S., see Birkmann, J.

Fekete, A., see Birkmann, J.

4 (2016) 1650013

1 (2016) 1671002

4 (2016) 1650017

4 (2016) 1650017

Fiedrich, F., see Birkmann, J.

4 (2016) 1650017

Fina, S., see Birkmann, J.

4 (2016) 1650017

Fleischhauer, M., see Greiving, S.

Forssén, K., see Molarius, R.

4 (2016) 1650014

4 (2016) 1650016

Fraser, A., Paterson, S. \& Pelling, M., Developing Frameworks to Understand Disaster Causation: From Forensic Disaster Investigation to Risk Root Cause Analysis

Friedrich, M., see Birkmann, J.

2 (2016) 1650008

4 (2016) 1650017

Garschagen, M., see Ajibade, I.

3 (2016) 1650009

Garschagen, M., see Birkmann, J.

4 (2016) 1650017

Garschagen, M., see Pelling, M.

3 (2016) 1650012

Garschagen, M., see Solecki, W.

3 (2016) 1650020

1 (2016) 1671001

Gerrard, M., see Sobel, A.

1 (2016) 1671002

Gibson, T. D., Pelling, M., Ghosh, A., Matyas, D., Siddiqi, A., Solecki, W., Johnson, L., Kenney, C., Johnston, D. \& Du Plessis, R., Pathways for Transformation: Disaster Risk Management to Enhance Resilience to Extreme Events

Goris, A., see Birkmann, J.

Goris, A., see Schwab, M.

1 (2016) 1671002

4 (2016) 1650017

4 (2016) 1650015

Greiving, S., Hurth, F., Hartz, A., Saad, S. \& Fleischhauer, M.,

Developments and Drawbacks in Critical Infrastructure and

Regional Planning: A Case Study on Region of Cologne,

Germany

4 (2016) 1650014

Greiving, S., see Birkmann, J.

4 (2016) 1650017 
Gurian, P., see Catalano de Sousa, M. R.

2 (2016) 1650006

Hall, T., see Sobel, A.

1 (2016) 1671001

Hallman, R., see Sobel, A.

1 (2016) 1671001

Handmer, J., The McMurray Fire

1 (2016) 1671003

Hartz, A., see Greiving, S.

4 (2016) 1650014

Hochrainer-Stigler, S., Mochizuki, J. \& Pflug, G., Impacts of Global and Climate Change Uncertainties for Disaster Risk Projections: A Case Study on Rainfall-Induced Flood Risk in Bangladesh

1 (2016) 1650004

Hurth, F., see Greiving, S.

4 (2016) 1650014

Jeffers, J. M., Hazards, Climates and Cultures: Reflections on

Recent Scholarship and Proposals for Further Research

2 (2016) 1602002

Johnson, L., see Gibson, T. D.

1 (2016) 1671002

Johnston, D., see Gibson, T. D.

1 (2016) 1671002

Keenan, J., see Sobel, A.

1 (2016) 1671001

Kenney, C., see Gibson, T. D.

1 (2016) 1671002

Kirshen, P. H., see Douglas, E. M.

4 (2016) 1650013

Kraines, S., see Nishi, M.

3 (2016) 1650011

Kuhlmann, U., see Birkmann, J.

4 (2016) 1650017

Kyne, D., A Fukushima-Like Disaster in New York City and

Possible Issues Associated with the Extreme Event Management System

Lall, U., see Sobel, A.

Leckner, M., see Mitchell, J. K.

Levy, M. A., see Sobel, A.

Ley, A., see Birkmann, J.

Link, H., see Solecki, W.

Lorenzoni, I., see Clarke, D.

Mäki, K., see Molarius, R.

Matyas, D., see Gibson, T. D.

Mauriello, M. N., Flood and Storm Hazard Adaptation in New

Jersey's Coastal Zone: Barriers and Opportunities

2 (2016) 1650007

1 (2016) 1671001

1 (2016) 1650003

1 (2016) 1671001

4 (2016) 1650017

3 (2016) 1650020

2 (2016) 1650010

4 (2016) 1650016

1 (2016) 1671002

Mcarthur, K., see Douglas, E. M.

2 (2016) 1671007

4 (2016) 1650013

McDermott, M., see Mitchell, J. K.

1 (2016) 1650003

4 (2016) 1650013

Mitchell, J. K., O’Neill, K., McDermott, M. \& Leckner, M.,

Towards a Transformative Role for Local Knowledge in Post-

Disaster Recovery: Prospects for Co-production in the Wake of

Hurricane Sandy

1 (2016) 1650003

Mitchell, J. K., Personal and Professional Encounters with

Hazards in Context: The Challenge of Ambiguity

2 (2016) 1671004 
Mitchell, J. K., see Birkmann, J.

4 (2016) 1650017

Mochizuki, J., see Hochrainer-Stigler, S.

1 (2016) 1650004

Molarius, R., Räikkönen, M., Forssén, K. \& Mäki, K., Enhancing the Resilience of Electricity Networks by Multi-Stakeholder

Risk Assessment: The Case Study of Adverse Winter Weather in Finland

Montalto, F. A., see Catalano de Sousa, M. R.

Murphy, C., see Clarke, D.

Narayanan, P., see Parasuram, P.

Niggol Seo, S., Untold Tales of Goats in Deadly Indian Monsoons: Adapt or Rain-Retreat Under Global Warming?

Nishi, M., Pelling, M., Yamamuro, M., Solecki, W. \& Kraines, S., Risk Management Regime and Its Scope for Transition in Tokyo

Novák, B., see Birkmann, J.

Nowak, W., see Birkmann, J.

Odunuga, S., see Birkmann, J.

O’Neill, K., see Mitchell, J. K.

Orlove, B., see Sobel, A.

Parasuram, P., Narayanan, P., Pelling, M., Solecki, W., Ramachandran, P. \& Ramachandran, R., Climate Change Adaptation Pathways in Kolkata

Paterson, S., see Fraser, A.

Pelling, M., Abeling, T. \& Garschagen, M., Emergence and

Transition in London's Climate Change Adaptation Pathways

Pelling, M., see Ajibade, I.

4 (2016) 1650016

2 (2016) 1650006

2 (2016) 1650010

3 (2016) 1650021

1 (2016) 1650001

3 (2016) 1650011

4 (2016) 1650017

4 (2016) 1650017

3 (2016) 1650019

1 (2016) 1650003

1 (2016) 1671001

Pelling, M., see Birkmann, J.

Pelling, M., see Fraser, A.

3 (2016) 1650021

2 (2016) 1650008

Pelling, M., see Gibson, T. D.

Pelling, M., see Nishi, M.

Pelling, M., see Parasuram, $\mathrm{P}$.

3 (2016) 1650012

3 (2016) 1650009

3 (2016) 1650019

2 (2016) 1650008

1 (2016) 1671002

3 (2016) 1650011

Pflug, G., see Hochrainer-Stigler, S.

3 (2016) 1650021

Räikkönen, M., see Molarius, R.

1 (2016) 1650004

Ramachandran, P., see Parasuram, P.

4 (2016) 1650016

Ramachandran, R., see Parasuram, P.

3 (2016) 1650021

3 (2016) 1650021

Rhyner, J., see Birkmann, J.

4 (2016) 1650017

Riegel, C., see Birkmann, J.

4 (2016) 1650017

Rilling, B., see Birkmann, J.

4 (2016) 1650017

Rosenzweig, C., see Sobel, A.

1 (2016) 1671001

Saad, S., see Greiving, S.

4 (2016) 1650014 
Schwab, M., Berchtold, C. \& Goris, A., An Integrated Critical Infrastructure Risk and Resilience Concept in the Context of Extreme Weather Events and Global Change

4 (2016) 1650015

Seager, R., see Sobel, A.

1 (2016) 1671001

Shaman, J., see Sobel, A.

1 (2016) 1671001

Siddiqi, A., see Gibson, T. D.

1 (2016) 1671002

Sobel, A., Camargo, S. J., Debucquoy, W., Deodatis, G., Gerrard, M., Hall, T., Hallman, R., Keenan, J., Lall, U., Levy, M. A., Orlove, B., Rosenzweig, C., Seager, R., Shaman, J. \& Tippett, M., Extreme Weather and Climate: Workshop Report

1 (2016) 1671001

Solecki, W., Link, H. \& Garschagen, M., Risk Management and Adaptation Transitions in New York City

Solecki, W., see Gibson, T. D.

3 (2016) 1650020

1 (2016) 1671002

Solecki, W., see Nishi, M.

3 (2016) 1650011

Solecki, W., see Parasuram, P.

3 (2016) 1650021

Stirling, D. A., A Historical Snapshot of the Zika Virus and

Concise Bibliography

1 (2016) 1650002

Thieken, A., see Birkmann, J.

4 (2016) 1650017

Tippett, M., see Sobel, A.

1 (2016) 1671001

Uitto, J. I., Environmental Hazards, Climate Change and Disaster

Risk Reduction: J. K. Mitchell's Relevance to the Global

Sustainable Development Agenda

2 (2016) 1671006

Ulbrich, U., see Birkmann, J.

4 (2016) 1650017

Vallée, D., see Birkmann, J.

4 (2016) 1650017

von Streit, J., see Birkmann, J.

3 (2016) 1650019

Watson, C., see Douglas, E. M.

4 (2016) 1650013

Welle, T. \& Birkmann, J., Measuring the Unmeasurable:

Comparative Assessment of Urban Vulnerability for Coastal

Megacities — New York, London, Tokyo, Kolkata and Lagos

3 (2016) 1650018

Welle, T., see Birkmann, J.

2 (2016) 1650005

Welle, T., see Birkmann, J.

3 (2016) 1650019

Welle, T., see Birkmann, J.

4 (2016) 1650017

Wenzel, F., see Birkmann, J.

4 (2016) 1650017

Wieprecht, S., see Birkmann, J.

4 (2016) 1650017

Yamamuro, M., see Nishi, M.

3 (2016) 1650011 